\title{
Dinitrohydrazines and Interaction of Them with Some Group-II Metals - DFT Treatment
}

\section{Lemi Türker}

Department of Chemistry, Middle East Technical University, Üniversiteler, Eskişehir Yolu No: 1, 06800 Çankaya/Ankara, Turkey; e-mail: 1turker@gmail.com; lturker@metu.edu.tr

\begin{abstract}
Dinitrohydrazines and interaction of them with some group-II metals have been considered within the restrictions of density functional theory and the basis set applied (B3LYP/6-311++G(d,p)). Dinitrohydrazine has two isomers as geminal and vicinal. The calculations reveal that both of them are structurally stable. The vicinal form electronically is more stable and thermo chemically more favorable than the other isomer. The beryllium magnesium and calcium (1:1) composites of them are considered. The results indicate that only the beryllium composites (geminal and vicinal) are structurally intact while the others undergo decomposition due to reductive cleavage by the metals. The decompositions occurred exhibit variations from one composite to the other.
\end{abstract}

\section{Introduction}

$\mathrm{N}$-nitrohydrazines have been somewhat neglected chemicals. However, Kalinin et al., managed to synthesize various $\mathrm{N}$-nitrohydrazines and their salts starting from the silylhydrazines and nitrating with nitrogen pentoxide [1]. Picard and Boivin reported the nitration of N,N'-disubstituted hydrazines [2]. The nitration of N,N'-disubstituted hydrazines by means of nitric acid/acetic anhydride was found to yield in most of the cases the azo derivatives. An exception was N,N'-dibenzoylhydrazine which under the same conditions gave N,N'-dinitro- N,N'-dibenzoylhydrazine [2]. On the other hand, Ball interested on the nitrohydrazines as potential high energy materials and performed a series of calculations to determine the optimized structures, vibrational frequencies, and fundamental thermodynamic properties of nitrohydrazine and dinitrohydrazine

Received: December 6, 2021; Accepted: January 4, 2022

Keywords and phrases: dinitrohydrazines; hydrazine; explosive; alkaline earth metals; nitramine.

Copyright (C) 2022 Lemi Türker. This is an open access article distributed under the Creative Commons Attribution License (http://creativecommons.org/licenses/by/4.0/), which permits unrestricted use, distribution, and reproduction in any medium, provided the original work is properly cited. 
employing G2, G3 methods, and two complete basis set [3]. The results of enthalpies of formation calculations suggest that nitrohydrazines are indeed high energy materials, with decomposition energies per gram exceeding ammonium nitrate and TNT but less than RDX or HMX.

\section{Method of Computation}

The initial geometry optimizations of all the structures leading to energy minima have been achieved by using MM2 method followed by semi-empirical PM3 self consistent fields molecular orbital (SCF MO) method [4,5] at the restricted level [6,7]. Subsequent optimizations were achieved at Hartree-Fock level employing various basis sets. Then, geometry optimizations were managed within the framework of density functional theory $[8,9]$ at the level of B3LYP/6-311++G(d,p) $[6,10]$. The exchange term of B3LYP consists of hybrid Hartree-Fock and local spin density (LSD) exchange functions with Becke's gradient correlation to LSD exchange [9,11]. The correlation term of B3LYP consists of the Vosko, Wilk, Nusair (VWN3) local correlation functional [12] and Lee, Yang, Parr (LYP) correlation correction functional [13]. The vibrational analyses also have been done. The total electronic energies are corrected for the zero point vibrational energy (ZPE). The normal mode analysis for each structure yielded no imaginary frequencies for the $3 N-6$ vibrational degrees of freedom, where $N$ stands for the number of atoms in the system. This has indicated that the structure of each molecule corresponds to at least a local minimum on the potential energy surface. All these calculations have been done by using the Spartan 06 package program [14].

\section{Results and Discussion}

\section{Dinitrohydrazines}

Dinitrohydrazine (DNH) is a nitramine type explosive and has two configurational forms; geminal and vicinal namely 1,1-dinitrohydrazine and 1,2-dinitrohydrazine. Figure 1 shows the optimized structures and the direction of dipole moment vectors of those.
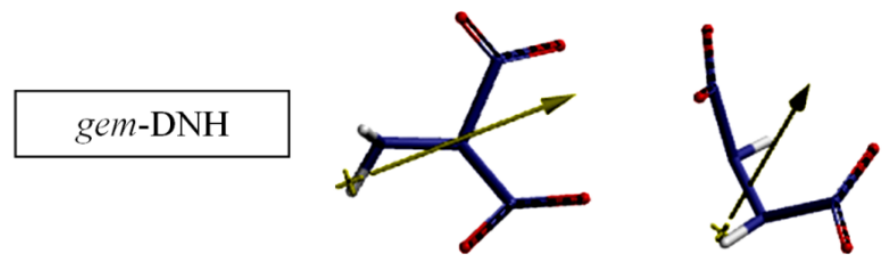

$v i c-\mathrm{DNH}$

Figure 1. Optimized structures of geminal and vicinal dinitrohdrazines. 
In the case of geminal form the dipole moment $(3.50 \mathrm{D})$ vector originates from somewhere around $\mathrm{NH}_{2}$ moiety of the hydrazine backbone towards the nitro groups. Note that the geminal dinitrohydrazine molecule is not a true push-pull type system because of the valency of the nitrogen atom bearing the two nitro groups. However, the lone pair of that nitrogen atom may delocalize over the nitro groups (acquiring itself some positive charge) even may be assisted by the lone pair of $\mathrm{NH}_{2}$ moiety ( $\alpha$-effect [15]). Consequently some of the $\sigma$-electron population of the N-N bond should shift to $\mathrm{N}\left(\mathrm{NO}_{2}\right)_{2}$ moiety, thus the $\mathrm{NH}_{2}$ group gets some positive charge as a whole (see Figure 2) (note that one of the nitro groups has more over all positive charge than the other one). Whereas the dipole moment of the vicinal isomer is $2.90 \mathrm{D}$, it is less than the geminal one due to opposing effects of $\mathrm{NNO}_{2}$ groups. Figure 2 shows the electrostatic potential (ESP) charges on the atoms of the dinitrohydrazines. Note that the ESP charges are obtained by the program based on a numerical method that generates charges that reproduce the electrostatic potential field from the entire wavefunction [14].
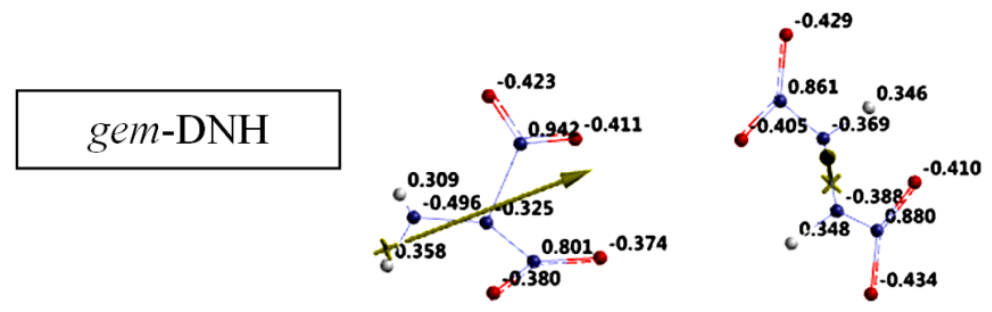

Figure 2. The ESP charges on atoms of the dinitrohydrazines.

Figure 3 shows the electrostatic potential maps of the dinitrohydrazines where the blue regions relatively stand for the most positive potential field and red ones, if exists any, the most negative ones.
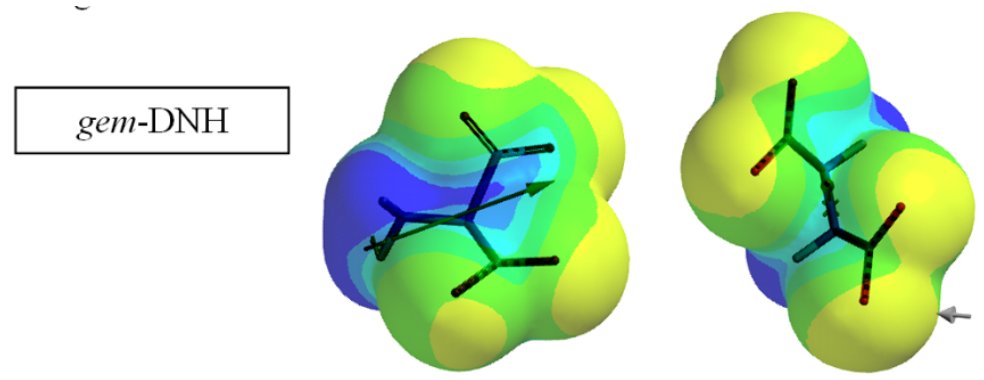

vic-DNH

Figure 3. Electrostatic potential maps of the dinitrohydrazines. 
Table 1 shows some energies of the dinitrohydrazines where $\mathrm{E}, \mathrm{ZPE}$ and $\mathrm{E}_{\mathrm{C}}$ stand for total electronic energy, zero point vibrational energy and the corrected total electronic energy, respectively. As seen in the table, of the two forms of dinitrohydrazine the vicinal isomer electronically is more stable than the geminal one.

Table 1. Some energies of the dinitrohydrazines.

\begin{tabular}{lccc}
\hline Structures & $\mathbf{E}$ & $\mathbf{Z P E}$ & $\mathbf{E}_{\mathbf{C}}$ \\
\hline gem $-\mathrm{DNH}$ & -1367840.61 & 145.83 & -1367694.78 \\
vic-DNH & -1367903.46 & 152.35 & -1367751.11 \\
\hline
\end{tabular}

Energies in $\mathrm{kJ} / \mathrm{mol}$.

Table 2 contains some standard state thermodynamic properties of the dinitrohydrazines. As seen in the table, the vicinal isomer is more exothermic and more favorable to form compared to the geminal one.

Table 2. Some thermodynamic properties of the dinitrohydrazines.

\begin{tabular}{llll}
\hline Structures & $\mathbf{H}^{\mathbf{o}}(\mathbf{k J} / \mathbf{m o l} \mathbf{)})$ & $\mathbf{S}^{\mathbf{0}}\left(\mathbf{J} / \mathbf{m o l}^{\mathbf{0}}\right)$ & $\mathbf{G}^{\mathbf{o}}(\mathbf{k J} / \mathbf{m o l} \mathbf{)})$ \\
\hline gem $-\mathrm{DNH}$ & -1367686.759 & 346.76 & -1367790.147 \\
vic-DNH & -1367744.746 & 336.02 & -1367844.933 \\
\hline
\end{tabular}

Figure 4 (see also Table 3) displays some of the molecular orbital energy levels of the dinitrohydrazines. The geminal isomer is characterized with higher HOMO but lower LUMO energies compared to the vicinal form. Consequently, the geminal one has much narrower frontier molecular orbital energy gap $(\Delta \varepsilon)$, thus should be more susceptible to impact stimulus because there exists an adverse correlation between the impact sensitivity and $\Delta \varepsilon$ value $[16,17]$.

Table 3 includes the HOMO, LUMO energies and the interfrontier molecular orbital energy gap $(\Delta \varepsilon)$ values $\left(\Delta \varepsilon=\varepsilon_{\mathrm{LUMO}}-\varepsilon_{\mathrm{HOMO}}\right)$ of the dinitrohydrazines. The vicinal isomer has lower HOMO but higher LUMO energy level. Note that in general electron donating substituents raise up both of the frontier molecular orbital energies whereas electron attracting ones lower the both energy levels. Conjugation on the other hand, raises up the HOMO, but lowers the LUMO energy levels [15]. In the dinitrohydrazines, these effects should be operating at different extends depending on the location of $\mathrm{NO}_{2}$ and $\mathrm{NH}_{2}$ groups. 


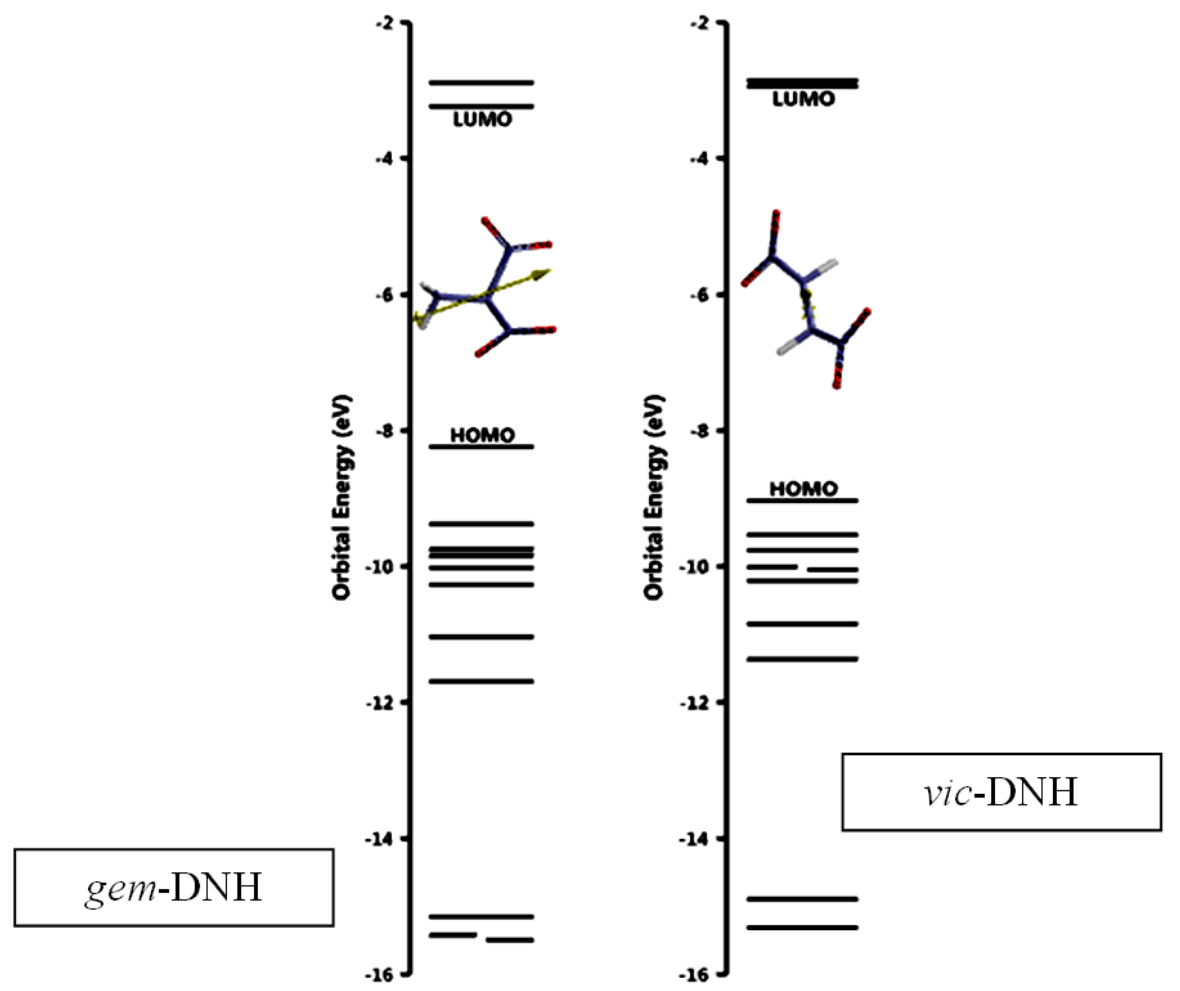

Figure 4. Some of the molecular orbital energy levels of the dinitrohydrazines.

Table 3. The HOMO, LUMO energies and $\Delta \varepsilon$ values of the dinitrohydrazines.

\begin{tabular}{lccc}
\hline Structures & HOMO & LUMO & $\boldsymbol{\Delta} \boldsymbol{\varepsilon}$ \\
\hline gem-DNH & -795.12 & -312.68 & 482.44 \\
vic-DNH & -871.07 & -283.56 & 587.51 \\
\hline
\end{tabular}

Energies in $\mathrm{kJ} / \mathrm{mol}$.

Figure 5 displays the HOMO and LUMO patterns of the dinitrohydrazines.

Figure 6 shows the local ionization potential maps of the dinitrohydrazines. In the local ionization potential map conventionally red regions on the density surface indicate areas from which electron removal is relatively easy, meaning that they are subject to electrophilic attack. On the other hand, regions having blue color represent areas where ionization is relatively difficult. 


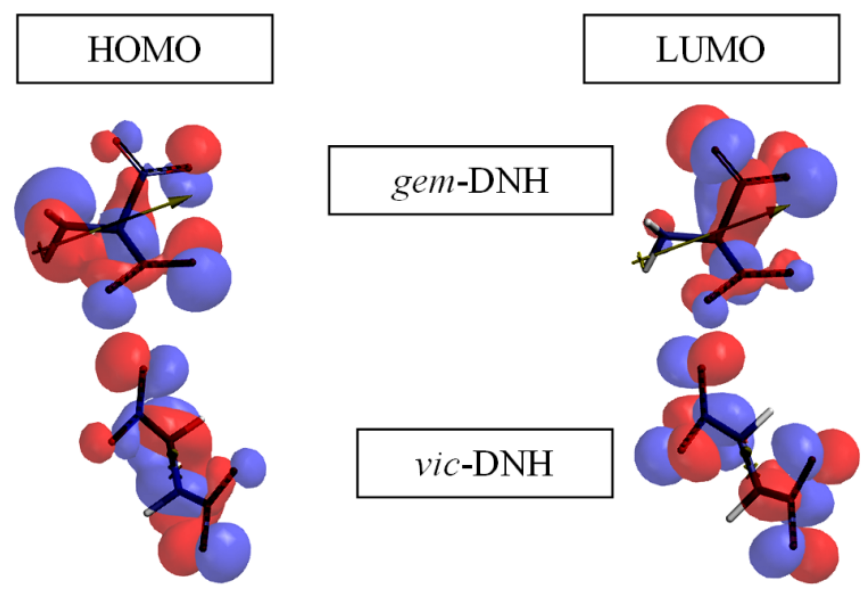

Figure 5. The HOMO and LUMO patterns of the dinitrohydrazines.
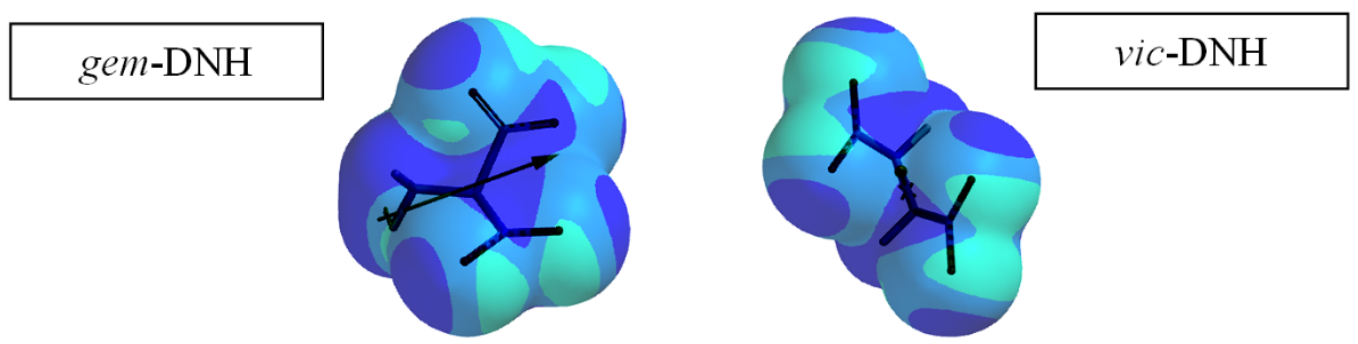

Figure 6. Local ionization potential maps of the dinitrohydrazines.

Figure 7 shows the LUMO maps of the dinitrohydrazines. A LUMO map displays the absolute value of the LUMO on the electron density surface. The blue color stands for the maximum value of the LUMO and the color red, the minimum value.
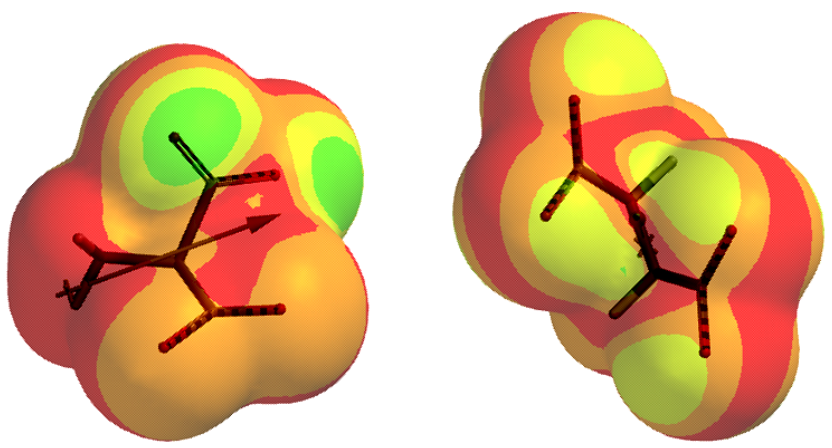

Figure 7. The LUMO maps of the dinitrohydrazines. 


\section{The metal composites of dinitrohydrazines}

Only, group-II metals of either $\mathrm{Be}, \mathrm{Mg}$ or $\mathrm{Ca}$ has been considered as the metallic component of the composite system. These alkaline-earth metals have different degrees of reducing power. As the counter part of the possible redox reactions, the dinitrohydrazines (DNH) stand for good candidates.

Figure 8 shows the optimized structures as well as the direction of the dipole moment vector of the DNH composite systems considered. However, note that most of the composites considered undergo decomposition. In the case of beryllium both of the geminal and vicinal isomers remain intact, contrary to magnesium and calcium cases.

Figure 9 shows the ESP charges on atoms of the DNH composites considered. The order of positive charges developed on the metal components are $\operatorname{Be}($ vic $)<\operatorname{Be}($ gem $)<$ $\operatorname{Mg}($ vic $)<\mathrm{Mg}($ gem $)<\mathrm{Ca}($ gem $)<\mathrm{Ca}($ vic $)$. The electron population transferred from metal to the most electrophilic site of the dinitrohydrazine moiety should shuffle electron distribution of the whole molecule and during the optimization process some bonds are ruptured to minimize the energy of the system.
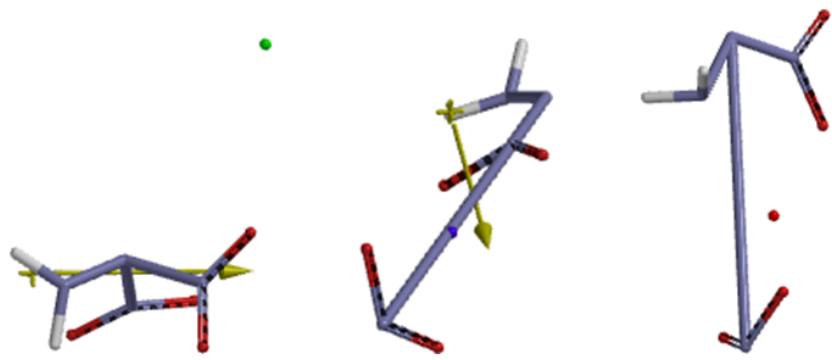

gem $-\mathrm{DNH}+\mathrm{Be}$

gem-DNH+Mg

gem-DNH+Ca
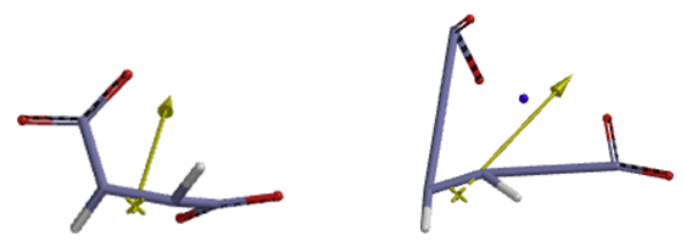

$v i c-\mathrm{DNH}+\mathrm{Be}$

vic- $\mathrm{DNH}+\mathrm{Mg}$

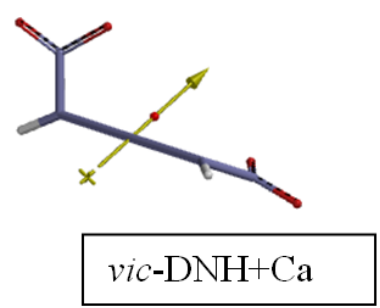

Figure 8. Optimized structures of the DNH composite systems considered. 

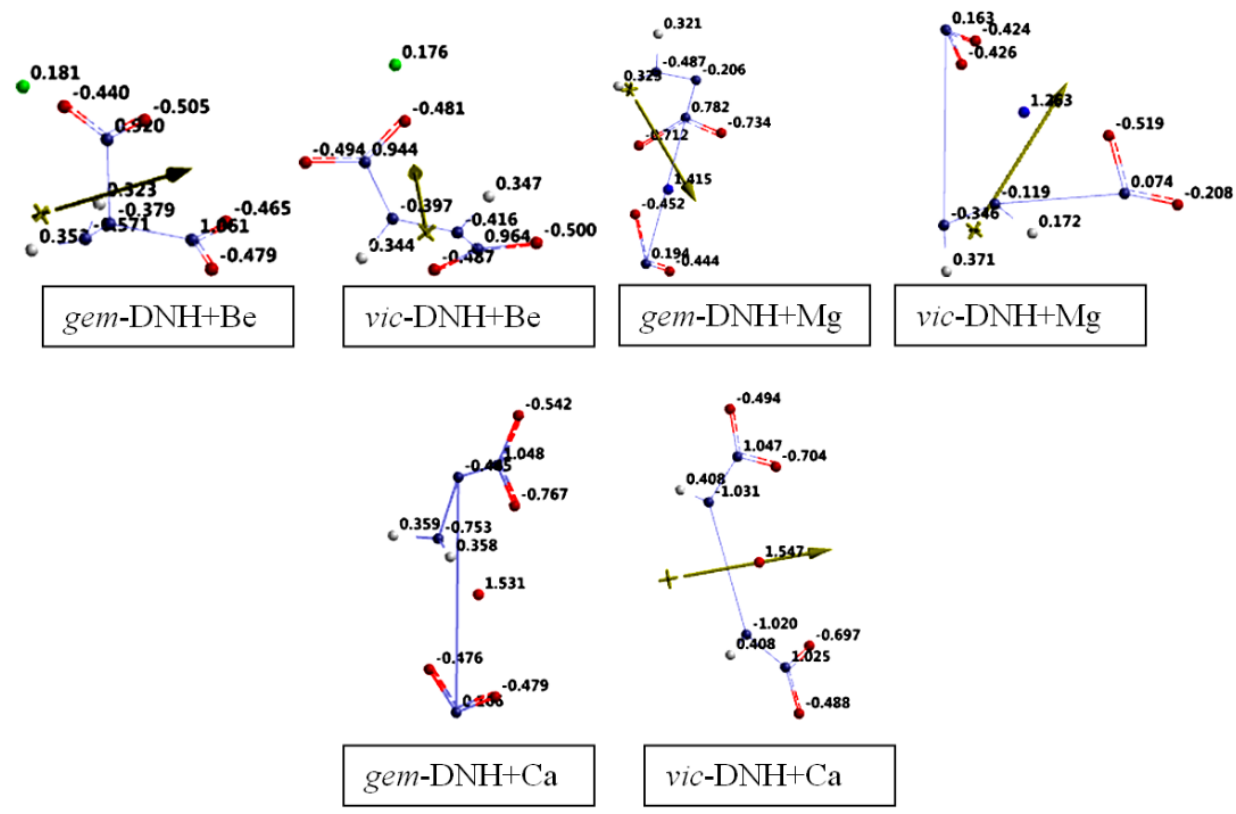

Figure 9. The ESP charges on atoms of the DNH composites considered.

Table 4 includes some energies of the composite systems considered. In the beryllium and calcium cases $v i c-\mathrm{DNH}+\mathrm{Be}$ and vic-DNH$+\mathrm{Ca}$ systems are electronically more stable than their corresponding geminal counterpart but this is not valid for the magnesium case. Of course, one must keep in mind that except the geminal and vicinal beryllium composites the rest are decomposed systems. The decomposition patterns are interesting. In the presence of magnesium $\mathrm{N}-\mathrm{NO}_{2}$ bond of the geminal isomer is broken only, but in the case of vicinal isomer both of the $\mathrm{NO}_{2}$ bonds undergo bond cleavage. Whereas in the calcium case, rupture of $\mathrm{N}-\mathrm{NO}_{2}$ bond of the geminal isomer but $\left(\mathrm{NO}_{2}\right) \mathrm{HN}-\mathrm{NH}\left(\mathrm{NO}_{2}\right)$ bond of the vicinal isomer is affected.

Table 4 . Some energies of the composites considered.

\begin{tabular}{lccc}
\hline & $\mathrm{E}$ & ZPE & $\mathrm{E}_{\mathrm{C}}$ \\
\hline gem-DNH+Be & -1406360.32 & 146.86 & -1406213.46 \\
vic $-\mathrm{DNH}+\mathrm{Be}$ & -1406423.05 & 152.39 & -1406270.66 \\
gem $-\mathrm{DNH}+\mathrm{Mg}$ & -1893718.32 & 146.82 & -1893571.5 \\
vic $-\mathrm{DNH}+\mathrm{Mg}$ & -1893677.31 & 135.73 & -1893541.58 \\
gem $-\mathrm{DNH}+\mathrm{Ca}$ & -3147421.24 & 142.60 & -3147278.64 \\
vic-DNH$+\mathrm{Ca}$ & -3147479.49 & 148.80 & -3147330.69 \\
\hline
\end{tabular}

Energies in $\mathrm{kJ} / \mathrm{mol}$. 
Table 5 contains the HOMO, LUMO energies and $\Delta \varepsilon\left(\Delta \varepsilon=\varepsilon_{\mathrm{LUMO}}-\varepsilon_{\mathrm{HOMO}}\right)$ values of the composite systems considered. The HOMO energy of gem-DNH+Be is lower than vic-DNH+Be. A similar behavior happens for the magnesium case. Whereas, in the calcium case the HOMO of vic-DNH+Ca is lower than gem-DNH+Ca. As for the LUMO energy order, vic-DNH+Mg $<$ gem $-\mathrm{DNH}+\mathrm{Be}<$ vic-DNH$+\mathrm{Be}<$ gem- $\mathrm{DNH}+\mathrm{Mg}<$ gem$\mathrm{DNH}+\mathrm{Ca}<v i c-\mathrm{DNH}+\mathrm{Ca}$. Consequently, $\Delta \varepsilon$ order becomes vic-DNH$+\mathrm{Mg}<$ gem$\mathrm{DNH}+\mathrm{Be}<v i c-\mathrm{DNH}+\mathrm{Be}<g e m-\mathrm{DNH}+\mathrm{Mg}<g e m-\mathrm{DNH}+\mathrm{Ca}<v i c-\mathrm{DNH}+\mathrm{Ca}$.

Table 5. The HOMO, LUMO energies and $\Delta \varepsilon$ values of the composites considered.

\begin{tabular}{lccc}
\hline Structure & HOMO & LUMO & $\boldsymbol{\Delta} \boldsymbol{\varepsilon}$ \\
\hline gem-DNH+Be & -602.70 & -306.96 & 295.74 \\
vic-DNH+Be & -600.68 & -283.72 & 316.96 \\
gem-DNH+Mg & -686.45 & -263.81 & 422.64 \\
vic-DNH+Mg & -671.28 & -404.02 & 267.26 \\
gem-DNH+Ca & -713.22 & -245.48 & 467.74 \\
vic-DNH+Ca & -784.90 & -195.21 & 589.69 \\
\hline
\end{tabular}

Energies in $\mathrm{kJ} / \mathrm{mol}$.

Some of the molecular orbital energy levels of DNH composite systems considered are depicted in Figure 10. Structurally intact composites (only DNH+Be isomers) exhibit quite similar molecular orbital energy spectra. The magnesium and calcium composites in which dinitrohydrazine molecule decomposed possess rather different molecular orbital energy spectra of the respective geminal and vicinal isomers, because of the different decomposition patterns thus the products. 

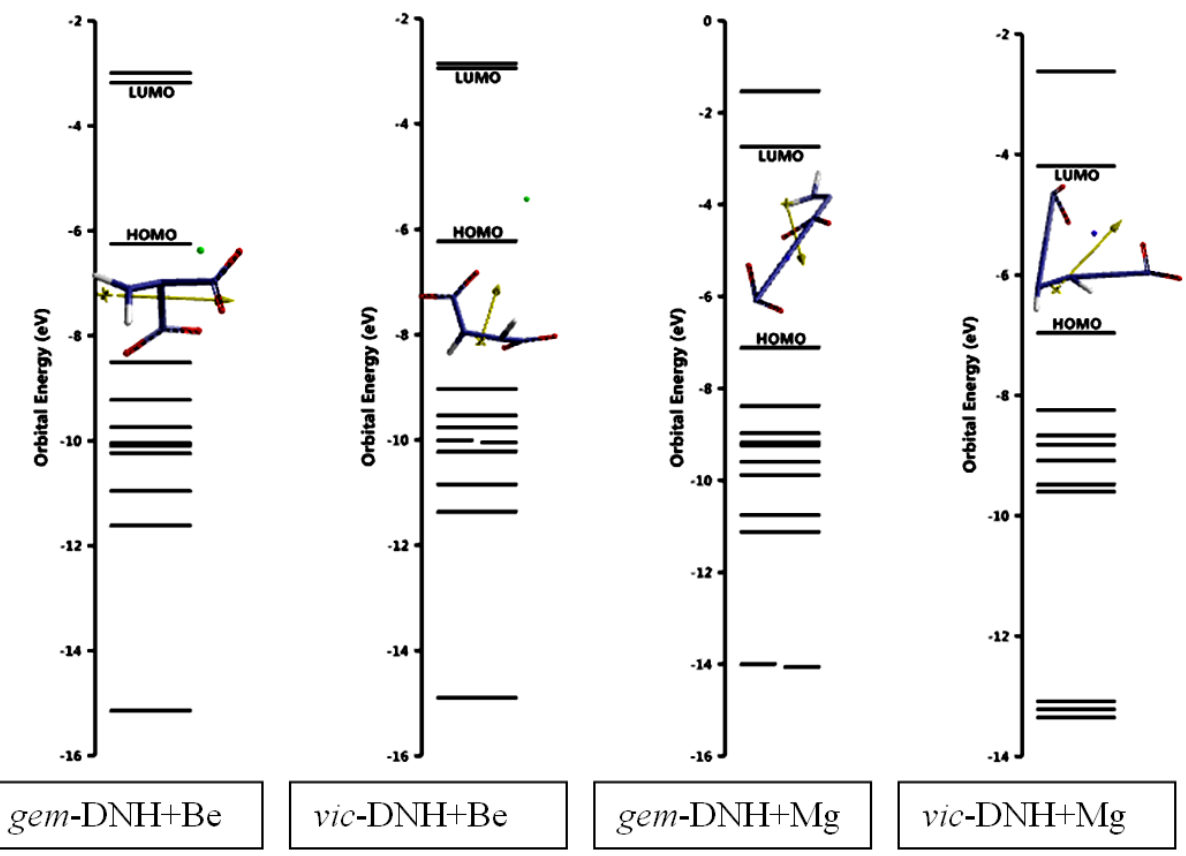

gem-DNH+Be
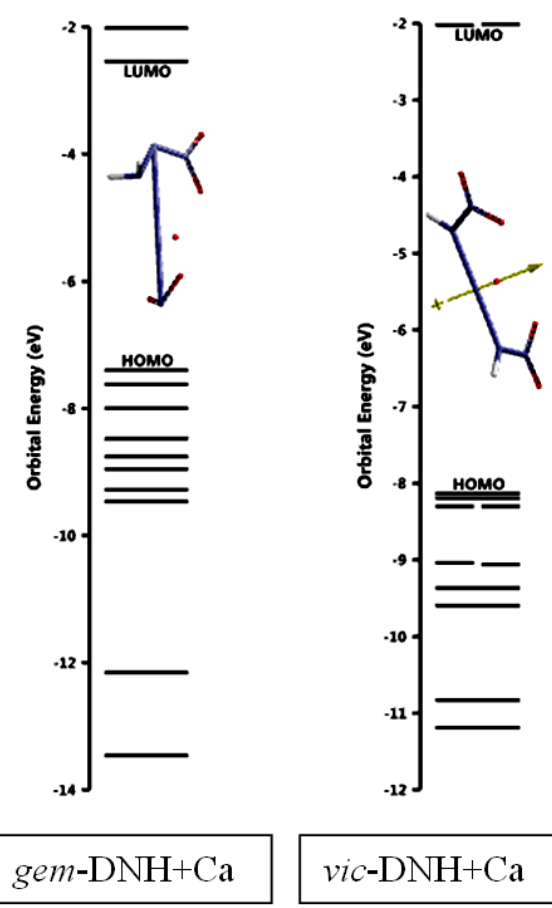

$v i c-\mathrm{DNH}+\mathrm{Ca}$

Figure 10. Some of the molecular orbital energy levels of DNH composites considered. 


\section{Conclusion}

In the present DFT treatise, within the constraints of the theory and the basis set employed, reveals that dinitrohydrazines are electronically stable and thermodynamically favorable. Of the two forms, the vicinal isomer is more stable and favorable than the geminal one. The beryllium composite is stable electronically and structurally intact but the magnesium and calcium composites are not so and dinitrohydrazine component undergoes decomposition either in the geminal or vicinal form. The magnesium composites exhibit $\mathrm{N}-\mathrm{NO}_{2}$ bond rupture. Although, only one of the $\mathrm{N}-\mathrm{NO}_{2}$ bond is affected in the geminal form, both of them are ruptured in the case of vic-DNH+Mg. In the calcium case, only one of the $\mathrm{NO}_{2}$ bond of the geminal isomer cleaves. Whereas, in the vicinal form $\left(\mathrm{NO}_{2}\right) \mathrm{HN}-\mathrm{NH}\left(\mathrm{NO}_{2}\right)$ bond undergoes bond rupture. All these reductive decompositions of dinitrohydrazines may open new horizons for the syntheses or conversions by extending the search to other group metals.

\section{References}

[1] Kalinin, A.V., Apasov, E.T., Ioffe, S.L., \& Tartakovskii, V.A. (1991). N-Nitrohydrazines and their salts. Izv. Akad. Nauk SSSR, Ser. Khim., 5, 1108-1114. https://doi.org/10.1002/chin.199250117

[2] Picard, J.P., \& Boivin, J.L. (1951). Study of nitration of N,N'-disubstituted hydrazines. Canadian J. Chem., 29, 223-227. https://doi.org/10.1139/v51-027

[3] Ball, D.W. (2006). Nitrohydrazines as potential high energy materials: High level calculations. Journal of Molecular Structure: THEOCHEM., 773, 1-7. https://doi.org/10.1016/j.theochem.2006.06.038

[4] Stewart, J.J.P. (1989). Optimization of parameters for semiempirical methods I. Method. J. Comput. Chem., 10, 209-220. https://doi.org/10.1002/jcc.540100208

[5] Stewart, J.J.P. (1989). Optimization of parameters for semi empirical methods II. Application. J. Comput. Chem., 10, 221-264. https://doi.org/10.1002/jcc.540100209

[6] Leach, A. R. (1997). Molecular modeling. Essex: Longman.

[7] Fletcher, P. (1990). Practical methods of optimization. New York: Wiley.

[8] Kohn, W., \& Sham, L. (1965). Self-consistent equations including exchange and correlation effects. J. Phys. Rev., 140, A1133-A1138. https://doi.org/10.1103/PhysRev.140.A1133

[9] Parr, R.G., \& Yang, W. (1989). Density functional theory of atoms and molecules. London: Oxford University Press. 
[10] Cramer, C.J. (2004). Essentials of computational chemistry. Chichester, West Sussex: Wiley.

[11] Becke, A.D. (1988). Density-functional exchange-energy approximation with correct asymptotic behavior. Phys. Rev. A, 38, 3098-3100.

https://doi.org/10.1103/PhysRevA.38.3098

[12] Vosko, S.H., Wilk L., \& Nusair, M. (1980). Accurate spin-dependent electron liquid correlation energies for local spin density calculations: a critical analysis. Can. J. Phys., 58, 1200-1211. https://doi.org/10.1139/p80-159

[13] Lee, C., Yang, W., \& Parr, R.G. (1988). Development of the Colle-Salvetti correlation energy formula into a functional of the electron density. Phys. Rev. B, 37, 785-789. https://doi.org/10.1103/PhysRevB.37.785

[14] SPARTAN 06 (2006). Wavefunction, Inc. Irvine, CA, USA.

[15] Fleming, I. (1976). Frontier Orbitals and Organic Chemical Reactions. London, Wiley.

[16] Anbu, V., Vijayalakshmi, K.A., Karunathan, R., Stephen, A.D., \& Nidhin, P.V. (2019). Explosives properties of high energetic trinitrophenyl nitramide molecules: A DFT and IM analysis. Arabian Journal of Chemistry, 12(5), 621-632.

https://doi.org/10.1016/j.arabjc.2016.09.023

[17] Badders, N.R., Wei, C., Aldeeb, A.A., Rogers, W.J., \& Mannan, M.S. (2006). Predicting the impact sensitivities of polynitro compounds using quantum chemical descriptors. Journal of Energetic Materials, 24, 17-33. https://doi.org/10.1080/07370650500374326 